\title{
GROUPS OF HOMEOMORPHISMS \\ AND \\ NORMAL SUBGROUPS OF THE GROUP OF PERMUTATIONS
}

\author{
P. T. RAMACHANDRAN \\ Department of Mathematics \\ Calicut University P.n. \\ Pin 673635 \\ Ind i a \\ (Received February 13, 1989)
}

ABSTRACT. In this paper, it is proved that no nontrivial proper normal subgroup of the group of permutations of a set $X$ can be the group of homeomorphisms of (X,T) for any topology $\mathrm{T}$ on $\mathrm{X}$.

KEY WORDS AND PHRASES.

1980 AMS SUBJECT CLASSIFICATION CODES.

1. INTRODUCTION.

The concept of the group of homeomorphisms of a topological space has been investigated by several authors for many years from different angles. Many problems relating the topological properties of a space and the algebraic propeties of its group of homeomorphisms were investigated. J. De Groot [1] proved that any group is isomorphic to the group of homeomorphisms of a topological space. A related, although possibly more difficult, problem is to determine the subgroups of the group of permutations of a fixed set $X$ which can be the group of homeomorphisms of (X, $T$ ) for some topology $T$ on $X$. This problem appears to have not been investigated so far. In this paper we prove a result in this direction. It is proved that no nontrivial proper normal subgroup of the group of permutations of a fixed set $X$ can be the group of homeomorphisms of $(X, T)$ for any topology $T$ on $X$. As a by-product we obtain a characterization of completely homogeneous spaces.

2. NOTATIONS AND RESULTS.

We denote the cardinality of a set $A$ by $|A| \cdot S(X)$ denotes the group of all permutations (bijections) of a set $X$. If $p$ is a permutation of a set $X$, then

$$
M(P)=\{x \in X: P(x) \neq x\}
$$


$A(X)$ denotes the group of all permutations of a set $X$ which can be written as a product of an even number of transpositions. If $\alpha$ is any infinite cardinal number,

$$
H_{\alpha}=\{p \in S(X):|M(p)|<\alpha\} \text {. }
$$

If $(X, T)$ is a topological space, the group of all homeomorphisms of (X,T) onto itself is called the group of homeomorphisms of $(X, T)$ and denotes by $H(X, T)$.

First we consider the case when $X$ is finite set.

LEMMA $: .$. . No nontrivial proper normal subgroup of the group $S(X)$ of permutations of a finite set $X$ can be the group of homeomorphisms of the topological space (X,T) for any topology $T$ on $X$.

PROOF. We can directly verify the result when $|x|<4$. When $|x|>5$, the only normal subgroups of $S(X)$ are the trivial subgroup, $A(X)$ and $S(X)$ itself. Let $T$ be a topology on $X$ such that $H(X, T)=A(X)$. Then $(X, T)$ is a homogeneous space since all 3cycles are in $A(X)$. Thus $(X, T)$ is a product of a discrete space and an indiscrete space by Ginsburg [2]. Then a partition $\left\{E_{i}\right\}_{i=1}^{n}$ of $X$ forms a basis for the topology $T$ on $X$. Here some $E_{i}$ contain at least two elements (say $a$ and $b$ ) for otherwise $T$ is discrete in which case $H(X, T)=S(X) \neq A(X)$, a contradiction. Now the transposition $(a, b)$ is a homeomorphism of $(X, T)$. This is again a contradiction for $(a, b) \neq A(X)$. Hence the result.

Now we proceed to extend the result to the case when $X$ is an infinite set.

We use the following lemma proved by Baer [3].

LEMMA 2.2. The normal subgroups of the group $S(X)$ of permutations of $X$ are precisely the trivial subgroup, $A(X), S(X)$ and the subgroups of $S(X)$ of the form $H$ for some infinite cardinal number $\alpha, \alpha<|x|$.

LEMMA 2.3. Let $X$ be any infinite set and $T$ any topology on $X$ such that $A(X)$ is a subgroup of a group of homeomorphisms of $(X, T)$. Then
a) $(X, T)$ is homogeneous.
b) Super sets of nonempty open sets of $(X, T)$ are open.
c) If $(X, T)$ is not indiscrete, every finite subset is closed (i.e. (X,T) is $\left.T_{1}\right)$.
d) If $(X, T)$ is not discrete, any two nonempty open sets intersect (1.e. $(X, T)$ is hyper-connected).
e) If $(X, T)$ is not discrete, no finite nonempty set is open.

PROOF. (a) Let $a$ and $b$ be two distinct points of $X$. Now choose two more distinct points $c$ and $d$ of $X$ other than $a$ and $b$. Consider the permutation $p=(a, b)(c, d)$. It is a homeomorphism of $(X, T)$ since it is an element of $A(X)$ and it maps a to b. Hence $(X, T)$ is homogeneous.

(b) Let $A$ be a nonempty open set of $(X, T)$ and $A \subset B \subset X$. If $A=X$ or $A=B$, the the result is evident. Otherwise choose an element $a$ of $A$ and $b$ of $B \backslash A$. Also choose 
two distinct points $c$ and $d$, other than $a$ and $b$, both from elther $A, B \backslash A$ or $X \backslash B$. Now the permutation $p=(a, b)(c, d)$ is a homeonorphism of $(X, T)$ since $p \in A(X)$. Then $A \cup\{b\}=A \cup p(A)$ is open. Thus

$$
B=\underbrace{U}_{b \in B \backslash A}(A \cup\{b\})
$$

is open. Hence the result.

(c) Since $(X, T)$ is not indiscrete, there exists a proper nonempty open set $A$ of $(X, T)$. Let $b \in X \backslash A$. Then $X \backslash\{b\}$ is open by $(b)$. Thus $\{b\}$ is closed. Then every singleton subset of $(X, T)$ is closed, since $(X, T)$ is homogeneous by (a). Hence every finite subset, being a finite union of singleton subsets is closed.

(d) Let $A$ and $B$ be two nonempty open subsets of $(X, T)$. Prove that $A \cap B \neq \phi$. Otherwise choose an element a from $A$ and $b$ from $B$. Here $a \neq b$. Choose two distinct points $c$ and $d$ other than $a$ and $b$ both from either $A$ or $B$ or $X \backslash(A \cup B)$. Now $p=(a, b)$ $(c, d)$ is a homeomorphism of $(X, T)$ since $p \varepsilon A(X)$. Then $\{b\}=p(A) \cap B$ is open. Since $(X, T)$ is homegeneous by $(a),(X, T)$ is discrete. This contradicts the hypothesis. Hence the result.

(e) If $(X, T)$ is indiscrete, the result is obvious. Otherwise every finite subset of $X$ is closed by (c). If a nonempty finite subset $F$ of $X$ is open, then both $F$ and $X \backslash F$ are open which contradicts (d). Hence the result.

REMARK 2.1. Lemma 2.3 shows that if $A(X)$ is a subgroup of $H(X, T)$, then $(X, T)$ is discrete or the nonempty open sets of $(X, T)$ form a filter.

LEMMA 2.4. Let $(X, T)$ be an infinite topological space in which nonempty open sets form a filter. Let $A$ be a proper closed subset of $(X, T)$. Then every permutation of $X$ which moves only the elements of $A$ is a homeomorphism of $(X, T)$.

PROOF. Let $p$ be a permutation of $X$ which moves only the elements of $A$. If $U$ is a nonempty open set,

$$
p(U) \supset U \cap(X \backslash A)
$$

and $U \cap(X \backslash A)$ is open and nonempty by hypothesis. Thus $p$ is an open map. Similarly we can prove that $p$ is a continuous map. Hence $p$ is a homeomorphism.

LEMMA 2.5. Let $(\mathrm{X}, \mathrm{T})$ be an infinite topological space which is neither discrete nor indiscrete such that the group of homeomorphisms $H$ of $(X, T)$ is a normal subgroup of $S(X)$ containing $A(X)$. If $K$ is a proper closed subset of $(X, T)$, then $|K|<|X|$.

PROOF. On the contrary let $|K|=|x|$. By the previous remark the nonempty open sets of $(X, T)$ form a filter. Let $p$ be any permutation of $X$ which moves every element of $\mathrm{K}$ and keeps every element of $\mathrm{X} \backslash \mathrm{K}$ fixed. Then by Lemma 2.4, $\mathrm{p}$ is a homeomorphism of $(X, T)$. Also $|M(p)|=|x|=|X|$. Then by Lemma 2.2, every permutation of $X$ is a homeomorphism of $(X, T)$ since $H$ is normal.

Without loss of generality we may assume that $|K|=|X| K \mid$ for otherwise take a suitable subset and that subset is also closed by Lemma 2.3. Now consider a 
permutation $t$ of $X$ which maps $K$ onto $X \backslash K$ and $X \backslash K$ onto $K$. Such a pernutation exists since $|K|=|X| K \mid$. Now $t$ is a homeomorphism of $(X, T)$ onto itself by the 1 ast paragragh. Hence $t(K)=X \backslash K$ is closed. Now both $K$ and $X \backslash K$ are open which contradicts Lemma 2.3. Hence the result.

LEMMA 2.6. Let $(X, T)$ be an infinite topological space which is neither discrete nor indiscrete such that the group of homeomorphisms $H$ of $(X, T)$ is a normal subgroup of $S(X)$ containing $A(X)$. Let $K$ be a proper closed subset of $(X, T)$. Then every permutation $p$ such that $|M(p)|<|K|$ is a homeomorphism and every subset $M$ of $X$ such that $|M|<|K|$ is closed.

PROOF. By the previous remark, the nonempty open sets in $(X, T)$ form a filter. Let $t$ be a permutation of $X$ which moves every element of $K$ and leaves every element of $X \backslash K$ fixed. Then by Lemma 2.4, $t \in H$ and $|M(t)|=|K|$. Then by Lemma 2.2, every permutation $p$ of $X$ such that $|M(p)|<|K|$ belongs to $H$ since $H$ is normal in $S(X)$.

Now prove that every subset $M$ of $X$ such that $|M|<|K|$ is closed. Without loss of generality, we may assume that $|M|=|K|$ for otherwise we can take a suitable subset of $K$ and the subsets of $K$ are also closed by Lemma 2.3. We have $|K|=|M|$ and $|X \backslash K|=|X \backslash M|$ since $|K|<|X|$ by Lemma 2.5. Then there exists a permutation $p$ of $X$ which maps $K$ onto $M, M$ onto $K$ and keeps every other element $f i x e d$. Then $|M(p)|<|K|+|M|$. If $K$ is finite, $M$ is also finite and hence closed by Lemma 2.3 . Therefore we may assume that $K$ is infinite. Then $|M(p)|<|K|+|M|=|K|$. Thus $p$ is a homeomorphism by the first paragraph. Then $M=p(K)$ is closed.

LEMMA 2.7. Let $(X, T)$ be an infinite topological space which is nefther discrete nor indiscrete such that the group of homeomorphisms $H$ of $(X, T)$ is a nontrivial normal subgroup of $S(X)$. Then $T=T_{\alpha}$ for some infinite cardinal number $\alpha$ such that $\alpha<|X|$ where

$$
T_{\alpha}=\{\phi\} \cup\{A \subset X:|X| A \mid<\alpha\}
$$

PROOF. Since $H$ is a nontrivial normal subgroup of $S(X)$, it contains $A(X)$ by Lemma 2.2. Then by Lemma 2.3 , every finite set is closed in $(X, T)$. Let

$$
\alpha=\operatorname{lnf}\{|B|: B \subset X, B \text { is not closed in }(X, T)\}
$$

Then $\alpha$ is an infinite cardinal number such that $\alpha<|x|$.

Now prove that $T=T_{\alpha}$. Here $T \subset T_{\alpha}$ for otherwise there exists $U \varepsilon T$ but $\mathrm{U} \in \mathrm{T}_{\alpha}$. Then $|\mathrm{X}| \mathrm{U} \mid>\alpha$. Now let $M$ be any subset of $\mathrm{X}$ such that $|M|=\alpha$, then $M$ is closed in $(X, T)$ by Lemma 2.6 since $X \backslash U$ is closed in $(X, T)$ and $|M|<|X \backslash U|$. This contradicts the definition of $\alpha$. Also $T_{\alpha} \subset \mathrm{T}$. For, if $A \in T_{\alpha}, A \neq \phi,|X \backslash A|<\alpha$, then $X \backslash A$ is closed in $(X, T)$ by the definition of $\alpha$. Thus $A \varepsilon T$. Hence the result.

LEMMA 2.8. The group of homeomorphisms of a topological space $(X, T)$, where $T$ is efther discrete, indiscrete or of the form

$$
T_{\alpha}=\{\phi\} \cup\{A \subset X:|X \backslash A|<\alpha\}
$$


for some infinite cardinal number $\alpha, \alpha \leq|X|$ is $S(X)$.

PROOF. Obviously the group of homeomorphisms of a discrete or indiscrete space coincides with the group of permutations.

Now let $p$ be a permutation of $X$ and $U$ be a nonempty open set in ( $X, T_{\alpha}$ ). Then $|X| U \mid<\alpha$. Then $|X \backslash p(U)|<\alpha$ since $p$ is a permutation. Thus $p(U)$ is open. Therefore $p$ is an open map. Similarly we can prove that $p$ is also continuous. Thus $p$ is a homeomorphism. Hence the result.

THEOREM 2.9. Let $X$ be an infinite set. Then no nontrivial proper normal subgroup of $S(X)$ can be the group of homeomorphisms of $(X, T)$ for any topology $T$ on $X$.

PROOF. Let $T$ be a topology on $X$ where the group of homeomorhisms of $(X, T)$ is a nontrivial normal subgroup of $S(X)$. Then $T$ is either discrete, indiscrete or of the form $\mathrm{T}_{\alpha}$ for some infinite cardinal number $\alpha<|\mathrm{X}|$ by Lemma 2.8 . Then $H(X, T)=S(X)$ by Lemma 2.8. Hence the result.

DEFINITION 2.1. A topological space $(X, T)$ is completely homogeneous if $H(X, T)=$ $S(X)$.

REMARK 2.2. It is not difficult to see that a finite completely homogeneous space is either discrete or indiscrete. We may use a method analogous to the proof of lemma 3(c). Larson [4] determined the completely homogeneous spaces. His result given below easily follows from the Lemmas 2.7 and 2.8 and the above remark.

THEOREM 2.10. A topological space $(X, T)$ is completely homogeneous if and only if the topology $T$ is either discrete, indiscrete or of the form

$$
\mathrm{T}_{\alpha}=\{\phi\} \cup\{A \subset \mathrm{X}:|\mathrm{X} \backslash \mathrm{A}|<\alpha\}
$$

for some infinite cardinal number $\alpha<|x|$.

ACKNOWLEDGEMENT.

The author is greatly indebted to Professor M. Rajagopalan for his help and encouragement during the preparation of this paper. Also he wishes to thank Professor T. Thrivikraman for his guidance. 
1. GROOT, DE J., Groups Represented by Homeomorphism Groups, Math..Annalen, 138 (1959), 80-102.

2. GINSBURG, J., A Structure Theorem in Finite Topology, Can. Math. Bu11 26(1), (1983), 121-122.

3. BAER, R., Die Kompositionsreihe der Gruppe Aller Eineindeutigen Abbildungen Einer Unendlichen Menge Auf Sich, Studia Math. 5, (1934), 15-17.

4. LARSON, R.E., Minimum and Maximum Topological Spaces, Bulletin De L'Academie Polonaise des Sciences, Serie des Sciences math., Astr. et phys., XVIII(12), (1970), 707-710.

5. KANNAN, V and RAJAGOPALAN, M., Constructions and Applications of Rigid Spaces III, Can. J. Math. XXX(5), (1978), 926-932.

6. KANNAN, V., Constructions and Applications of Rigid Spaces I, Advances in Mathematics, 29(1), (1978), 89-130.

7. KANNAN, V. and RAJAGOPALAN, M., Constructions and Applications of Rigid Spaces II, American Journal of Mathematics, 100(6), (1978), 1139-1172. 


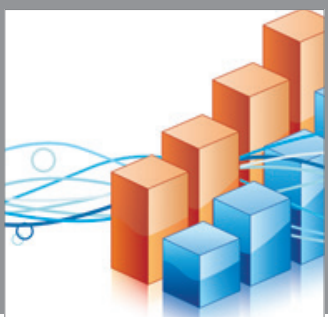

Advances in

Operations Research

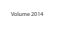

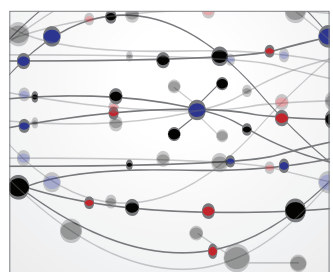

\section{The Scientific} World Journal
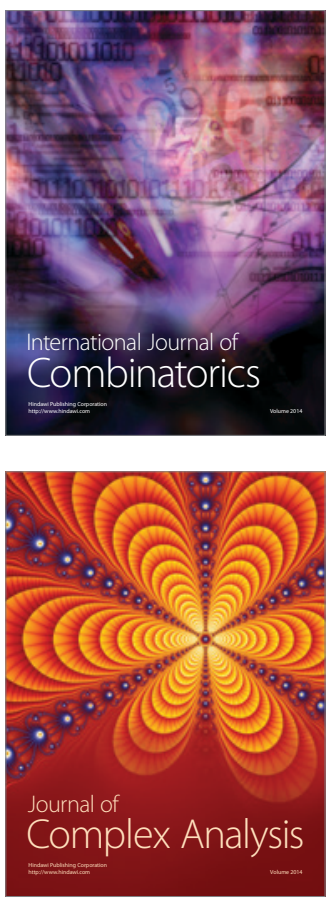

International Journal of

Mathematics and

Mathematical

Sciences
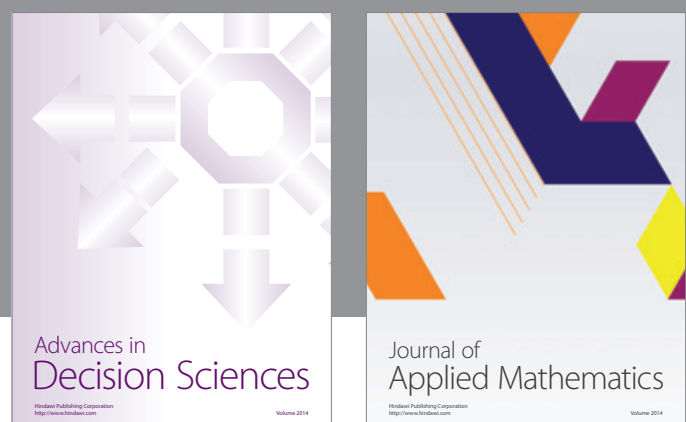

Journal of

Applied Mathematics
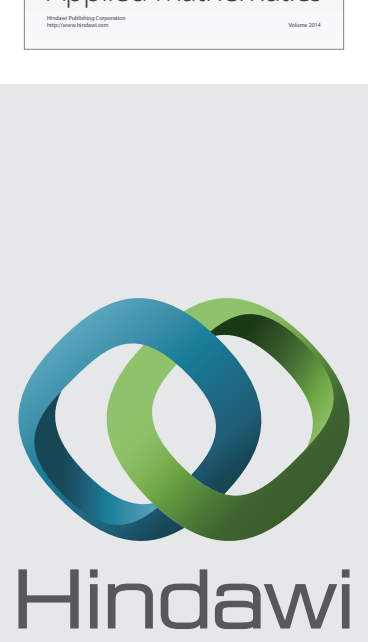

Submit your manuscripts at http://www.hindawi.com
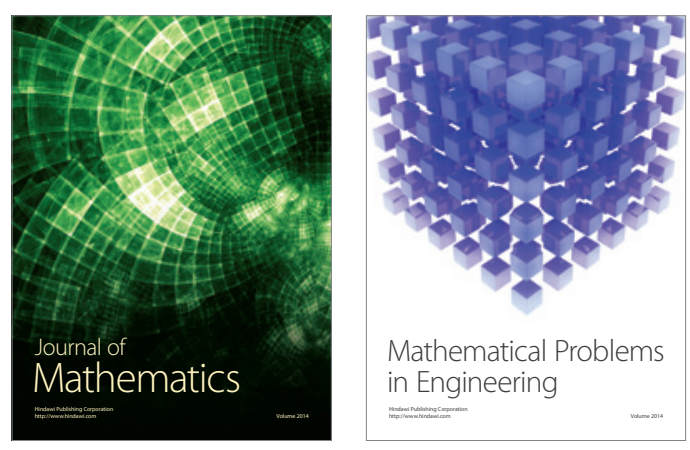

Mathematical Problems in Engineering
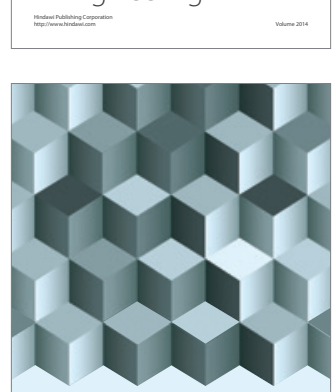

Journal of

Function Spaces
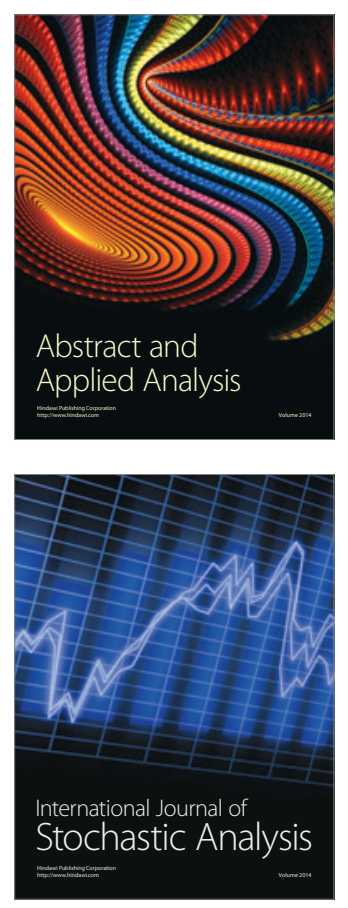

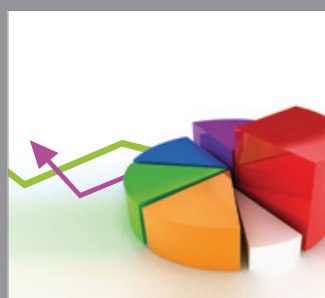

ournal of

Probability and Statistics

Promensencen
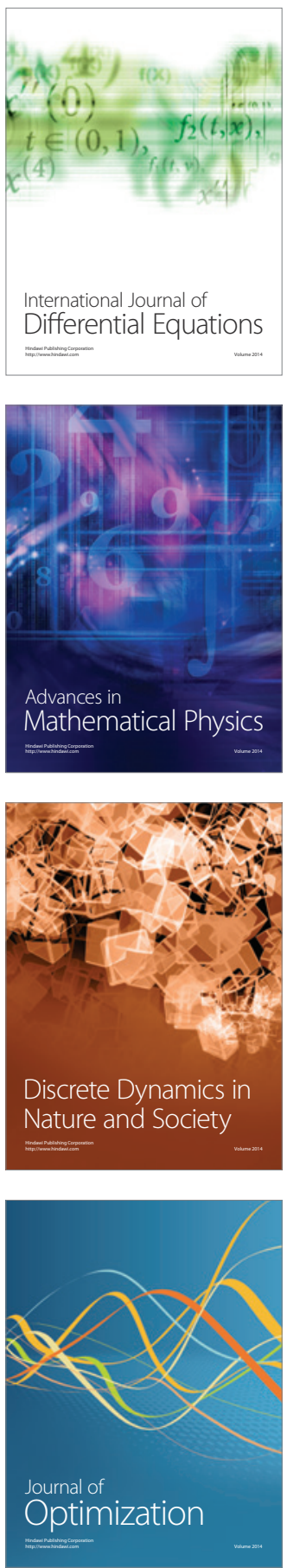